\title{
Extinction and the necessity or non-necessity of anticipating reward on nonrewarded trials ${ }^{1}$
}

E. J. CAPALDI, Purdue University, Lafayette, Ind. 47907, and D. R. ZIFF and R. C. GODBOUT, University of Texas, Austin, Texas 78112

Rats receiving nonrewarded trials but not previous rewarded trials showed a PRE. These data are similar to those produced in INE studies, except that fewer nonrewards were employed here. The results are not consistent with the hypothesis that at least one rewarded trial must precede nonrewarded trials in order to obtain a PRE, nor do they support the idea that reward must be expected on nonrewarded trials in order to obtain a PRE. Reinforcing this view are the results of studies that did not attempt to eliminate expectancy, as here, but rather manipulated its strength prior to extinction.

According to one approach (e.g., Amsel, 1958), a PRE is dependent upon expecting reward on nonrewarded trials, such expectancy being itself dependent upon an unspecified but moderate number of prior rewarded trials. This view was successfully challenged by McCain (1966), who demonstrated that a PRE could occur following a small number of partial-reinforcement trials. McCain, whose findings have proven replicable (e.g., Godbout, Ziff, \& Capaldi, 1968; Padilla, 1967), concluded that resistance to extinction ( $R$ to $E$ ) could be increased by nonrewarded trials on which reward is not expected. Subsequently, the frustration position was modified such that $R$ to $E$ could be increased by nonrewarded trials, provided these were preceded by a minimum of one rewarded trial (Amsel, Hug, \& Surridge, 1968). Thus, while it is still maintained that $R$ to $E$ will be increased only by nonrewarded trials on which reward is expected, it is now assumed that $r_{g}$ will develop much more rapidly than previously supposed.

However, it has frequently been demonstrated that even when the single "indispensable" reward is omitted, $\mathrm{R}$ to $\mathrm{E}$ will nevertheless be increased by nonrewarded trials (McCain, 1966; Robbins, Chait, \& Weinstock, 1968; Spear, Hill, \& O'Sullivan, 1965; Spear \& Spitzner, 1967). However, Amsel et al (1968), in presenting their hypothesis, did not cite these investigations, which suggests obviously that they do not consider them relevant to their view. Perhaps this is because these studies employed either a considerable number of nonrewarded trials in the experimental phase or nonrewarded preexperimental alley exploration. Thus some, or perhaps all, of these studies may not be appropriately described as small-trial studies. Spear and Spitzner (1967) have referred to the increased $R$ to E produced in these studies as the initial nonreward effect, or INE. Whether the INE and the small-trial PRE are similar or different remains to be determined.

The present investigation may be conceptualized either as a small-trials PRE study with prior reward omitted or as an INE study employing a small number of nonrewarded trials. The purposes of this study were several, among them to test the one-reward hypothesis of Amsel et al (1968) under clearly appropriate experimental conditions and to determine if the small-trials PRE and the INE might be treated on the same conceptual basis. As in previous INE studies, the number of nonrewarded trials was varied, being either two or five.

\section{METHOD}

Seventy-eight 90-day-old male rats, obtained from the Holtzman Co., Madison, Wisconsin, were run in a straight-alley runway identical to that employed by Capaldi \& Capaldi (1970). The runway included 14-in. start, 52-in. run, and 16-in. goal sections. A guillotine door allowed confinement of the $S$ in the goal section.

During the 21-day pretraining phase, Ss were handled every day, adjusted to a $12 \mathrm{-g}$ daily ration, and given experience eating .045 -g Noyes pellets in the home cage. In order to dissociate handling and feeding, the $E$ who always handled the $S$ s was ungloved, while the second $E$, who always fed Ss their daily ration in the home cage, wore surgical gloves. The Ss were never fed less than $30 \mathrm{~min}$ following handling, were never handled at feeding time, and were never fed at handling time.

The experimental phase consisted of 1 day of acquisition training followed by 1 . day of extinction training. Reward (R) was $24 \quad .045-\mathrm{g}$ pellets; nonreward $(\mathrm{N})$ was $30 \mathrm{sec}$ of confinement in the unbaited goalbox. Group NS $(\mathrm{N}=26)$ was trained NNRR, i.e., the first two acquisition trials were nonrewarded, the second two rewarded. Group NL $(\mathrm{N}=26)$ was trained NNNNNRR. Group RS $(\mathrm{N}=13)$ was given four rewarded trials, and Group RL $(N=13)$ was given seven rewarded trials. The amount fed in the runway was subtracted from the $12 \mathrm{~g}$ daily ration.
On the day following acquisition, 10 extinction trials were given to all Ss. During both acquisition and extinction, $S$ s were run in squads of six, two from Group NS, two from Group NL, and one each from Groups RS and RL. The intertrial interval varied between 6 and $8 \mathrm{~min}$. If $\mathrm{S}$ remained in a particular alley section for $60 \mathrm{sec}$, it was gently forced into the goalbox, and a time score of $60 \mathrm{sec}$ was assigned for that section and subsequent sections.

It should be mentioned that all Ss received one rewarded goalbox placement during acquisition. For half the $S s$ in Groups NS and NL, a rewarded placement occurred following the last nonrewarded trial, the remaining half of the Ss in these groups receiving a rewarded placement between the two rewarded trials. This variable is ignored here both because it was not a significant source of variation in any comparison and because the specific placement procedure employed made it difficult, if not impossible, to relate the variables to current accounts of placement effects.

\section{RESULTS}

Speed (reciprocated time) differences between the groups were not significant either on Trial 1 of acquisition or on Trial 1 of extinction. Figure 1 shows mean speeds in each alley section for each group on Trial 1 of extinction and on the remaining nine trials of extinction in blocks of three trials. Comparison of Groups NS and NL vs Groups RS and RL indicated that mean extinction speed over the 10 extinction trials was significantly increased by experience with nonrewarded acquisition trials only in the goal section $(F=5.87, \quad$ df $=1 / 74, \quad p<.05) . \quad A$ corresponding Trials by Groups analysis indicated that experience with nonrewarded acquisition trials resulted in an increased rate of extinction in the start section $(F=2.07, p<.05)$, a reduced rate of extinction in the run section $(F=2.63$, $\mathrm{p}<.01$ ), and a greatly reduced rate of extinction in the goal section $(F=4.15$, $\mathrm{p}<.001 ; \mathrm{df}=9 / 666$ in all cases). An orthogonal comparison, involving Groups NS vs NL, revealed that differences in mean extinction speed were greatest, but nevertheless not significant, in the goal section $(F=2.75, \quad d f=1 / 74, p>.10)$. Group NL demonstrated a slower rate of extinction than did Group NS, with this difference increasing from start $(F=1.57$, n.s.) through run $(F=1.67, p<.10)$ to goal $(F=2.03, p<.05 ; \mathrm{df}=9 / 666$ in all cases). None of the comparisons involving Groups RS vs RL approached significance. The present results are consistent with those of previous small-trials PRE investigations (e.g., Godbout et al, 1968) in 

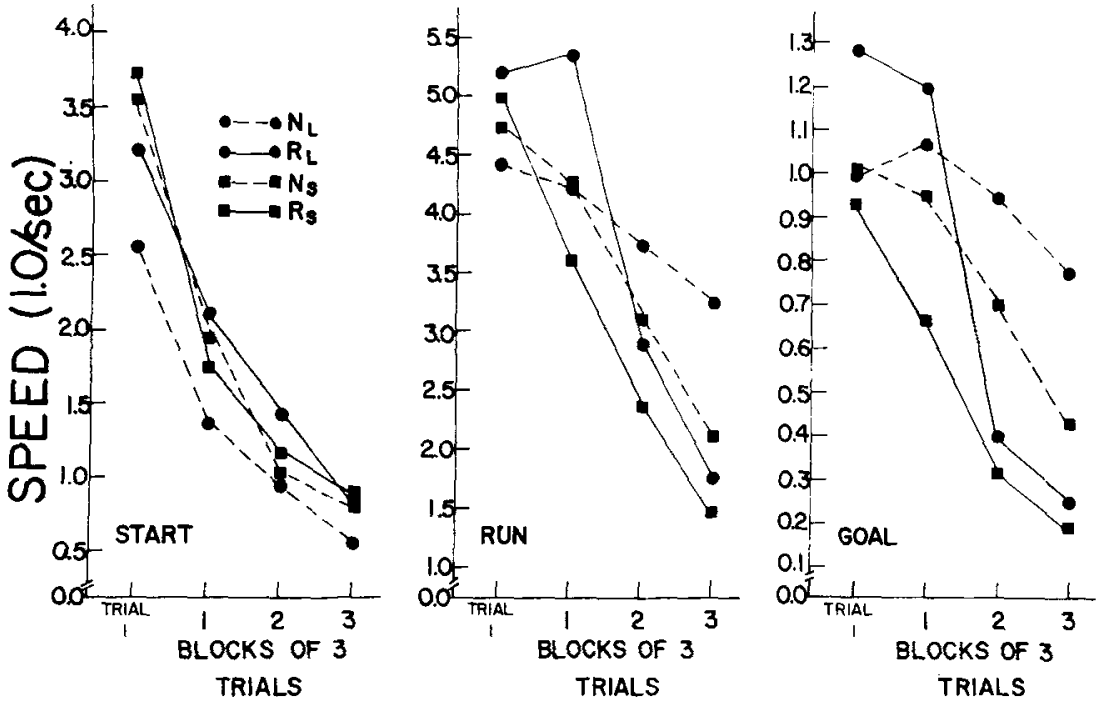

showing that experience with nonrewarded acquisition trials led to differences that became increasingly larger from start through goal.

\section{DISCUSSION}

The finding of a PRE here, or small-trials INE, provides little support for the proposition that $R$ to $E$ is increased only by nonrewarded trials on which reward is expected. Certainly, if reward were expected here, the source of that expectancy cannot be a prior rewarded trial, as Amsel et al (1968) maintained, since, of course, reward did not precede nonreward in this investigation. As to other sources of $r_{g}$, consider that whenever the animal was removed from the home cage in pretraining, it was not fed. Thus, the daily home-cage removal would hardly have been a cue for $I_{g}$. Moreover, on being returned to the home cage, feeding never occurred fewer than $30 \mathrm{~min}$ after and by a different E than had removed the Ss. ${ }^{2}$ The experimental apparatus was new to the animal, and $S s$ had not been trained previously in any other. Similar precautions were observed in INE studies, e.g., see Spear \& Spitzner (1967). McCain's hypothesis that $R$ to $E$ will be increased by nonrewarded trials on which reward is not expected is therefore tenable.

It may be assumed, of course, that some residual $\mathrm{r}_{\mathrm{g}}$ (source presently unspecified) was operative here. Relevant to this assumption would be the results of investigations which varied $\mathrm{I}_{\mathrm{g}}$ (rather than attempting to eliminate it as here), with the object of determining if such increases in expectancy were accompanied by increased $R$ to $E$. If a positive relationship of this sort were demonstrated, the assumption that some residual expectancy was operative here would be tempting. Much evidence, however, is quite unfavorable to the idea of a positive relation between $r_{g}$ and $R$ to $E$. For example, in a partial-reward investigation which equated number of nonrewarded trials (e.g., 72), but varied number of rewarded trials $(36,72$, or 144$), R$ to $E$ failed to increase as number of rewards increased but rather remained constant (Lawrence \& Festinger, 1962). Such groups presumably had the same number of "primary frustration experiences." But as number of rewards increased, an increase should have occurred both in the intensity of these frustrative experiences $\left(r_{g}\right.$ is assumed to be an increasing function of number of rewards; see Hug \& Amsel, 1969) and in the number of opportunities for conditioning $s_{f} \rightarrow R_{A P P}$, and thus, $R$ to $E$ should have increased. But $R$ to $E$ remained constant. This phenomenon seems unrelated to training level since the same results were produced when number of nonrewards was 27 , number of rewards being either 15 or 27 , and when number of nonrewards was 8 , number of rewards being 8,15 , or 32.3 Quite related to these findings are the results of studies that varied magnitude of partial reward. While early magnitude studies (Hulse, 1958; Wagner, 1961) are consistent with the hypothesis of a positive relation between $r_{g}$ and $R$ to $E$, later ones (e.g., Capaldi, in press; Leonard, 1968) have shown that, in some instances, as reward magnitude and thus $r_{g}$ increased, $R$ to $E$ decreased rather than increased. All of these magnitude findings and others as well can be interpreted within a sequential framework (e.g., Capaldi, in press) which, as yet, has not made any assumption concerning the role of expectancy on $\mathrm{R}$ to $\mathrm{E}$. Within a sequential framework, such an assumption is considered premature at this time. Also, data are available (E.g., Capaldi \& Capaldi,
Fig. 1. Mean extinction speed for the four experimental groups on the first trial of extinction and on the remaining nine extinction trials, in blocks of three trials.

1970; Grosslight \& Radlow, 1957) that suggest that expecting a larger reward and receiving a smaller reward cannot be both a necessary and a sufficient condition for increasing $R$ to $E$. In such investigations, certain groups received smaller reward or nonreward when presumably they should have been expecting larger reward but, nevertheless, failed entirely to show a PRE. On the basis of the three classes of findings considered above, the relationship between expectancy and $R$ to $E$, if any, seems a far more complex one than has thus far been recognized.

Here, as in the INE investigations that employed a greater number of nonrewards, $\mathrm{R}$ to $\mathrm{E}$ was increased by nonreward. Also, $\mathbf{R}$ to $\mathbf{E}$ increased here as number of nonrewards increased. This finding was reported by Robbins et al (1968), but Spear and his associates reported that $R$ to $E$ was independent of number of nonrewards. In any event, the sequential hypothesis (e.g., Capaldi, 1966) would ascribe the increased $R$ to $E$ accompanying the increased number of nonrewards to a variable called $\mathrm{N}$-length. The present results suggest that $\mathrm{N}$-length produces the same effects (more or less) early in training as late in training. In this connection, note, from a sequential standpoint, the consequences of the assumption that the nonrewarded stimulus that is neutral early in training (i.e., $\mathrm{S}^{\mathrm{N}}$ ) becomes, with additional training, frustrative or disappointing (i.e., SNF). Groups given small numbers of acquisition trials would have $S^{N}$ conditioned to the reaction, while those given more extensive training would have SNF conditioned to the reaction. But extinction nonrewards following limited training would produce the neutral stimulus (i.e., $\mathrm{S}^{\mathrm{N}}$ ), while extinction nonrewards following extensive training would produce a stimulus containing the disappointment component (i.e., SNF). How the addition of the disappointment component to $S^{N}$ would affect $R$ to $E$ would depend upon whether its presence in extinction increased, decreased, or left unaltered the generalization decrement produced by $\mathrm{SN}^{\mathrm{N}}$ itself. However, before deciding which of the three general possibilities mentioned is most feasible, it must first be decided whether or not, with increased training, $\mathrm{S}^{\mathrm{N}}$ becomes $\mathrm{S}^{\mathrm{NF}}$ REFERENCES

AMSEL, A. The role of frustrative nonreward in noncontinuous reward situations. Psychological Bulletin, 1958, 55, 102-119.

AMSEL, A., HUG, J. J., \& SURRIDGE, C. $\Upsilon$. Number of food pellets, goal approaches, and 
the partial reiuforcement effect after minimal acquisition. Journal of lixperimenta Psychology, 1968, 77, 530-534.

CAPALDI, E. J. Partial reinforcement: An hypothesis of sequential effects. Psychological Review, 1966, 73, 459-477.

CAPALDI, E. J. An analysis of the role of reward and reward magnitude in instrumental learning. In J. H. Reynierse (Ed.), Current issues in animal learning. Lincoln: University of Nebraska Press, in press.

CAPALDI, E. J., \& CAPALDI, E. D. A discrepancy between anticipated reward and obtained reward with no increase in resistance to extinction. Psychonomic Science, 1970, 18, 19-21.

GODBOUT, R. C., ZIFF, D. R., \& CAPALDI, E. J. Effect of several reward exposure procedures on the small trial PRE. Psychonomic Science, 1968, 13, 153-154.

GROSSLIGHT, J. H., \& RADLOW, R. Patterning effect of the nonreinforcement-reinforcement sequence involving a single nonreinforced trial. Journal of Comparative \& Physiological Psychology, 1957, 50, 23-25.

HUG, J. J., \& AMSEL, A. Frustration theory and partial reinforcement effects: The acquisition-extinction paradox. Psychological Review, 1969, 76, 419-421.

HULSE, S. H. Amount and percentage of reinforcement and duration of goal confinement in conditioning and extinction. Journal of Experimental Psychology, 1958, $56,48-57$.

LAWRENCE, D. H., \& FESTINGER, L. Deterrents and reinforcement. Stanford: Stanford University Press, 1962.

LEONARD, D. W. Amount and sequence of reward in partial and continuous reinforcement. Journal of Comparative \& Physiological Psychology, 1969, 67, 204-211.

McCAIN, G. Partial reinforcement effects following a small number of acquisition trials. Psychonomic Monograph Supplements, 1966, 1 (Whole No. 12), 251-270.

PADILLA, A. M. A few acquisition trials: Effects of magnitude and per cent reward. Psychonomic Science, 1967, 9, 241-242.

\section{Types of Pavlovian conditioning procedures used in establishing $\mathrm{CS}+$ and their effect upon}

avoidance behavior

DENNIS K. KAMANO, Galesburg State Research Hospital, Galesburg, Ill. 61401

The purpose of this study was to determine the effects on avoidance responding of superimposing fear stimuli $(C S+)$ established by Pavlovian delay and trace conditioning. Coterminous delay conditioning resulted in an accelerated avoidance-response rate, but the effect of CSt offset was not clear, since the suppression effect expected for CSt offset did not occur in all Ss. On the other hand, a CS+ established by trace conditioning enhanced avoidance responding both during and after presentation.
ROBBINS, D., CHAIT, H., \& WIIINSTOCK, S. Effects of nonreinforcement on running behavior during acquisition, extinction, and reacquisition. Journal of Comparative \& Physiological Psychology, 1968, 66, 699-706. SPEAR, N. F., HILL, W. I., \& O'SULLIVAN, D. 1. Acquisition and extinction after initial trials without nonreward. Journal of Experimental Psychology, 1965, 69, 25-29.

SPEAR, N. E., \& SPITZNER, J. H. Effect of initial nonrewarded trials: Factors responsible for increased resistance to extinction. Journal of Experimental Psychology, 1967, 74 525-537.

WAGNER, A. R. Effects of amount and percentage of reinforcement and number of acquisition trials on conditioning and extinction. Journal of Experimental Psychology, 1961, 62, 234-242.

\section{NOTES}

1. This investigation was supported in part by National Institute of Child Health and Human Development Research Grant HD 04379 to the first author and by Public Health Service Fellowships 1-F1-MH-38,131-02 and 2-F1-MH-32,183-04 from the National Institute of Mental Health to the second and third authors, respectively.

2. Incidental evidence suggested that there was little or no tendency for the Ss to expect reward upon home cage removal or handling. Although the $E$ (surgical gloves) who fed the $S s$ in the home cage noticed general excitement, including scratching and biting, the second $\mathbf{E}$ (ungloved) who handled the Ss noted a lack of excitement and a general docility.

3. The findings considered above, it has been suggested by Lawrence and Festinger, are favorable to their dissonance position. It is difficult to understand, however, why dissonance theory, no less than frustration theory (and more or less for the same reasons), would not expect $R$ to $E$ to increase as number of rewards increased with number of nonrewards being held constant (number of dissonant experiences equated, but intensity of thesc varied due to greater expectation). avoidance relative to the baseline rate (Herendeen \& Anderson, 1968; Kamano, 1968; Rescorla, 1967a). On the other hand, a CSt established by Pavlovian trace conditioning may not exert such an antagonistic effect. Relatively short CS-offset/US-onset intervals in trace-conditioning procedures produce a CSt whose presentation also enhances avoidance but does not suppress avoidance-response rate following offset (Rescorla \& LoLordo, 1965).

The purpose of the present study was to repeat the experiments of delay and trace conditioning with a control procedure added to provide a baseline from which the effects of these conditioning procedures could be evaluated. This study, using rats as Ss, involved a direct comparison within a single $S$ of the three procedures.

\section{SUBJECTS}

The Ss were three 60-day-old male Wistar albino rats from the colony maintained at the Galesburg State Research Hospital and were housed in individual cages throughout the experiment. APPARATUS

The apparatus was a modified Mowrer-Miller shuttlebox, with a 10.16-cm-high hurdle and a drop gate that, when lowered, prevented crossing. A $10-\mathrm{W}$ light bulb, mounted above each compartment, served as the CS, and shock was delivered through a grid floor. Details of the apparatus and shock source are described elsewhere (Kamano, 1968).

\section{PROCEDURE}

Previous studies of the interaction between Pavlovian and instrumental avoidance conditioning (e.g., Rescorla, 1967; Rescorla \& LoLordo, 1965) served to guide the procedure used in this study.

Avoidance training, Pavlovian conditioning (alternating with avoidance training), and a test period were given in sequence to all Ss. This entire training sequence was repeated for each of the three Pavlovian procedures; however, the three different Pavlovian training conditions (delay, trace, and Superimposing a CS associated with a random-control) were administered to each random conditioning procedure resulted in $\mathrm{S}$ in a different order. Thus, for each $\mathrm{S}$, the near base-rate avoidance performance.

It has been demonstrated repeatedly that Sidman avoidance response rate can be altered by the presentation of a fear stimulus (CSt) established by Pavlovian conditioning procedures. The effects on response rate may, however, vary as a function of the temporal relation between the CS and US used in establishing the CSt. A CSt, established by coterminous Pavlovian delay conditioning, enhances the avoidance-response rate during presentation, while its offset depresses sequence and order of training was as follows: $S A$, avoidance training (AT)-random control (RC)-test (T) first, then AT-delay conditioning (DC)-T, then AT-trace conditioning (TC)-T. S B, AT-TC-T first, then AT-DC-T, then AT-RC-T. SC, AT-DC-T first, then AT-RC-T, then AT-TC-T.

Avoidance Training

The $S$ s received shuttlebox avoidance training on a Sidman avoidance schedule, with a shock-shock interval of $5 \mathrm{sec}$, response-shock interval of $30 \mathrm{sec}$, and shock intensity of 450 microamperes. One 